
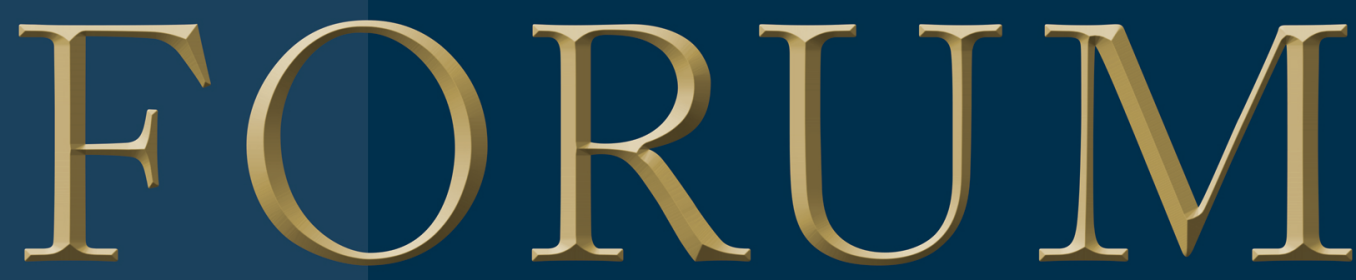

VOLUME 31 | NUMBER 3 MAY/JUNE

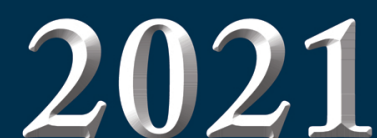

HAIR TRANSPLANT FORUM INTERNATIONAL

IN THIS ISSUE

Effect of Controlled Breathing on Pain Tolerance during Local Anesthesia at Donor Site in HT Strip Surgery

Hair Science Mini-Series: A "Hairy" Excursion into the HypothalamicPituitary-Thyroid Axis

New Motorized Handpiece to Improve Follicular Unit Excision

Personalizing Medicine for Hair Loss Using TrichoTest

\section{PLAN TO ATTEND!}

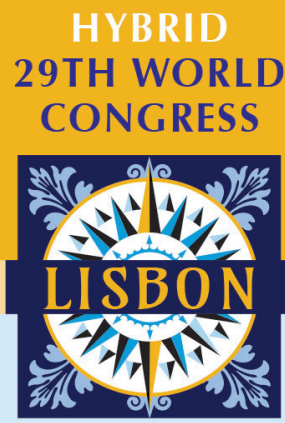

October 21-23, 2021

29thannual.org

Don't Miss

The Big One!

INTERNATIONAL SOCIETY OF HAIR RESTORATION SURGERY

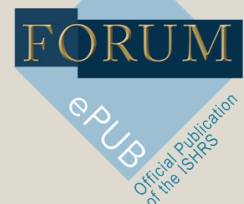

\title{
New Strategies for Using Grafts Harvested from Outside the Safe Zone
}

\author{
Robert H. True, MD, MPH, FISHRS I New York, New York USA I drtrue@hairlossdoctors.com
}

\section{ABSTRACT}

In contemporary hair restoration practice, harvesting grafts outside of the traditional safe donor zone has become commonplace. This occurs because of the need to avoid overharvesting, to homogenize the appearance of the donor, and to obtain the maximal lifetime donor grafts with follicular unit excision (FUE). In the majority of practices, grafts harvested from outside the safe zone are not treated any differently than those harvested from inside the safe zone, despite being more likely to be dihydrotestosterone (DHT) sensitive and to not survive as long as those from inside the safe zone when transplanted. ${ }^{1}$ The author proposes several strategies to intentionally place grafts harvested from outside the safe zone that optimize the utility of these grafts and minimize potential problems created by their use.

Keywords: dihydrotestosterone, follicular unit excision, safe donor zone

This article is open access and may not be copied, distributed, or modified without written permission from the International Society of Hair Restoration Surgery.

\section{INTRODUCTION}

With the emergence of follicular unit excision (FUE) as the predominant modality of graft harvesting in hair restoration surgery around the world, harvesting outside the traditional safe zone has become routine. This happens for three main reasons:

1. In a majority of cases, to get more than 2,500 grafts in a single surgery, the surgeon must harvest outside the traditional safe zone to avoid overharvesting (Figure 1).

2. In order for the donor area to look natural, particularly when the hair is worn short, the surgeon

FIGURE 1. Two examples of large harvests of $\pm 3,000$ in which harvesting was very wide and included not only the safe zone but also the upper and lower intermediate zones.
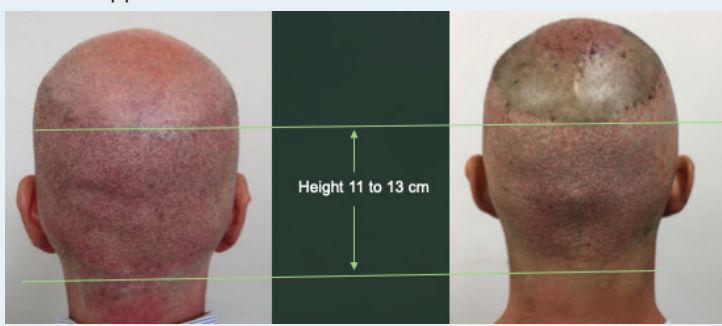
must harvest above and below the safe zone to create a homogenous appearance (Figure 2).

FIGURE 2. (A) A patient with native low density. Difference in density in harvested safe zone and above and below is not so evident, but with another harvest it would be evident. (B) The cosmetic problem when FUE harvesting is confined to the safe zone only. (C) The homogenized cosmetic appearance when the harvesting is extended above and below the safe zone.
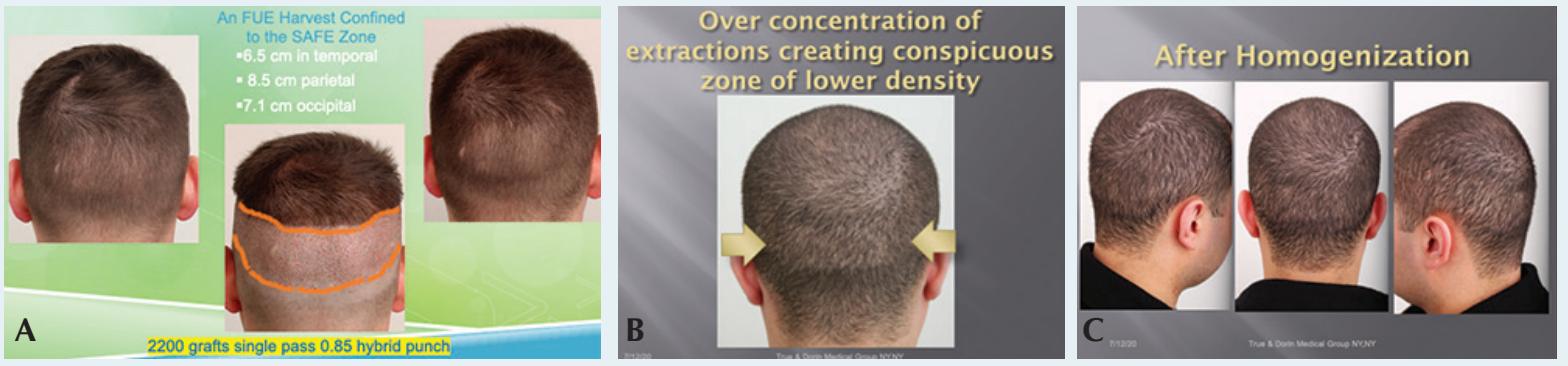
FIGURE 3. Patient who had 8,000 grafts harvested by FUE without evidence of overharvesting because the harvest included the intermediate zones.

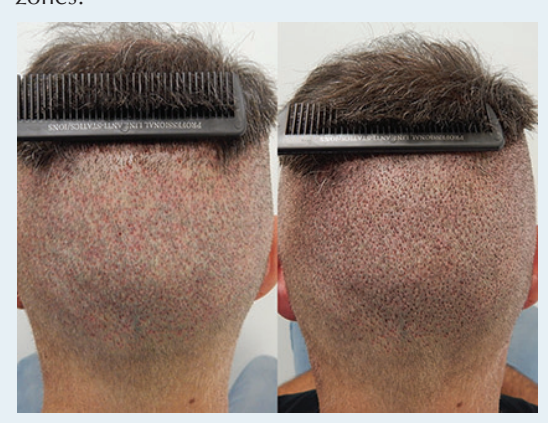

3. In patients who are having their entire restoration done with FUE, the donor zone must be enlarged to obtain a large number of grafts beyond the approximate 6,000 that can usually be harvested otherwise (Figure 3).

For many patients, $25 \%$ or more of their grafts will come from FIGURE 4. A harvest in which almost $50 \%$ of the grafts come outside the from outside of the safe zone. Marked area for harvesting (left) traditional and pattern of actual harvest (right).
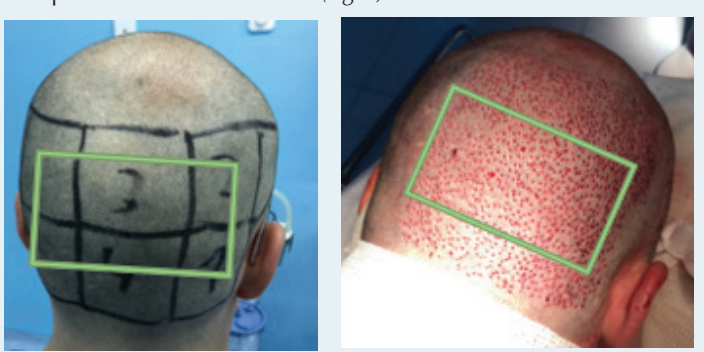

safe zone

(Figure 4). In the majority of practices, grafts harvested from outside the safe zone do not receive any particular attention or treatment. They are harvested, stored, and placed without regard for their higher potential to be dihydrotestosterone (DHT) sensitive. If these grafts end up being placed in a concentrated manner, close together, odd look-

FIGURE 5. Consequences of harvesting the intermediate zones as balding progresses.
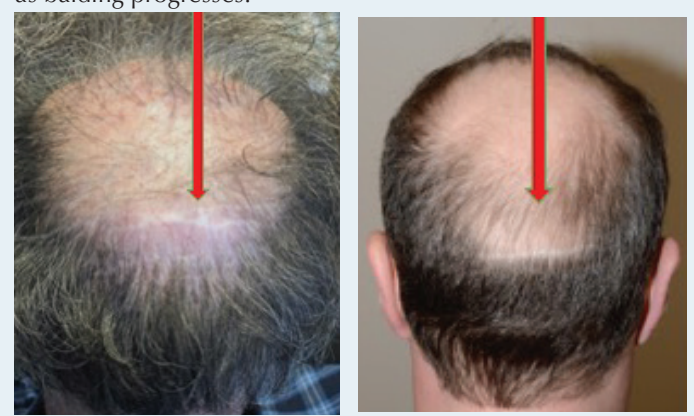

ing patches of balding could potentially develop, which might present significant cosmetic issues for some patients (Figure 5).

My interest in this topic was inspired by

Dr. Sebastian Yrariat's concept used in reconstructive scalp surgery: Potentially Androgenic Hair Transplantation (PAHT). ${ }^{2}$

\section{A NEW CONCEPT FOR SCALP DONOR ZONES}

In androgenetic alopecia (AGA), the follicles on the sides and back of the head in the safe zone are the most resistant to DHT-mediated balding. The author proposes that there are also two intermediate zones-one above the safe zone and one below - that have follicles that may or may not be DHT sensitive (Figure 6), and these two intermediate zones may undergo balding in some patients. Several studies (summarized in Figure 7) have indicated that 10\%-20\% of AGA patients will have class 7 baldness in their 60s, going up to $26 \%$ in their 80 s. The prevalence of retrograde alopecia is not well documented, but probably does not affect more than $5 \%-10 \%$ of those with AGA. If the intermediate zones go bald, over time, the grafts harvested from them will stop producing hair earlier than they would if taken from within the safe zone (Figure 8).

\section{Risk Assessment}

There are several factors to consider that help to identify patients at risk for loss of hair from the intermediate zones:

- Those who had early onset of AGA in the late teens or early 20s.

- Those who have many male relatives with class $6 / 7$ baldness.

- Those who have increased miniaturization in the intermediate zones (more than $15 \%)$.

- Young patients who will not or cannot use finasteride/minoxidil long term.

In my opinion, preservation of transplanted hair is another indication for long-term medical treatment. I realize this statement goes against convention FIGURE 7. Table showing the probability of developing severe balding.

\begin{tabular}{|l|l|l|l|l|}
\hline Physician & Pationts & Age & Pattom & $\%$ \\
\hline Norwood & 156 & $50-59$ & VII & 3 \\
\hline Norwood & 149 & $60-69$ & VII & 10 \\
\hline Norwood & 102 & $70-79$ & VII & 11 \\
\hline Norwood & 77 & Over 80 & VII & 17 \\
\hline Unger & 11 & $65-69$ & VII & 20 \\
\hline Unger & 12 & $70-74$ & VII & 14.8 \\
\hline Unger & 10 & $75-79$ & VII & 13.7 \\
\hline Unger & 31 & Over 80 & VII & 26.1 \\
\hline
\end{tabular}

FIGURE 8. Loss of the hair in the upper and lower intermediate zones in advanced balding.

and is provocative. Nevertheless, probable loss of hair from grafts harvested from the intermediate zones is a good indication for long-term use of finasteride and minoxidil. I think it is our responsibility to explain to our patients that some of the harvested grafts (as much as 25\%?) may stop producing hair earlier. Long-term use of minoxidil and finasteride will help to preserve follicles harvested and transplanted from the intermediate zones.

When we identify patients at risk for balding in the intermediate zones, we need to have clear strategies on when and where we place intermediate zone grafts such that we avoid creating cosmetic problems arising from transplanted hair that stops growing prematurely (Figure 9).

The follicles are often different in the two intermediate zones. In the upper intermediate zone, the hairs tend to be thicker and have higher hairs per follicular unit; in the lower zone, the hairs are finer and have fewer hairs per follicular unit. 
FIGURE 9 Donor area showing signs of instability.
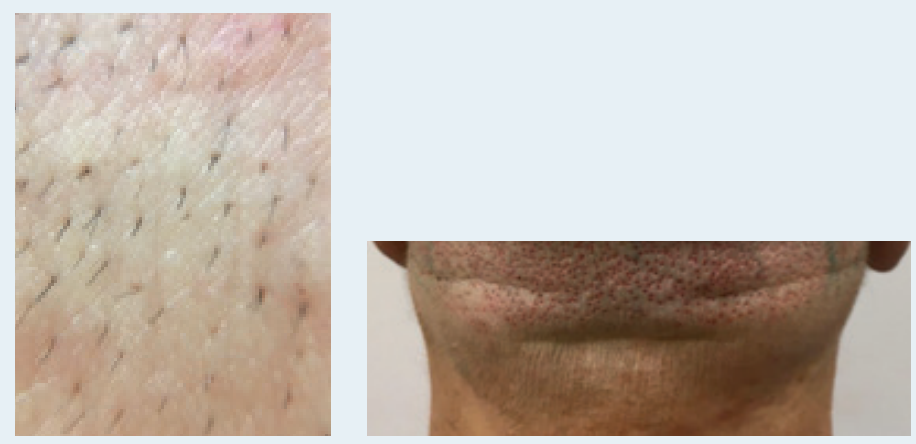

Graft Storage According to Harvest Zone

In order to pursue intentional strategies for use of grafts harvested outside of the safe zone, the grafts must be identified during storage and placement.
FIGURE 10. Storage option 1 in which the grafts from each zone are stored in separate

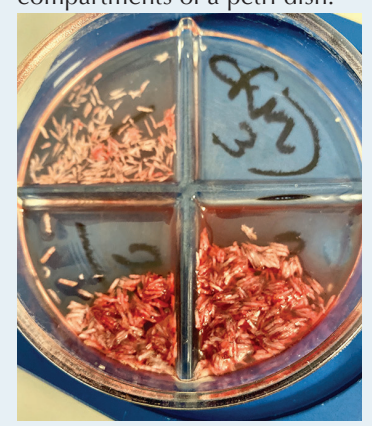
compartments of a petri dish.
Option 1: Use a divided petri dish to store the grafts from each zone in separate compartments regardless of hairs per graft (Figure 10).

Option 2: Store the grafts from each zone in separate petri dishes and place the grafts according to hairs/graft into different compartments (Figure 11).

The second option is the most detailed and offers the most versatility for employing different strategic placing strategies.
FIGURE 11. Storage option 3 in which the grafts from each zone are stored in separate petri dishes and then sorted by number of hairs.

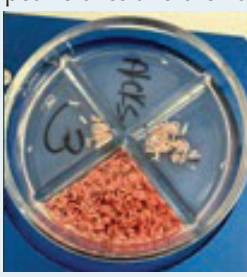

Lower intermediate zone

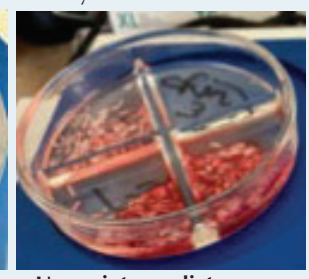

Upper intermediate zone

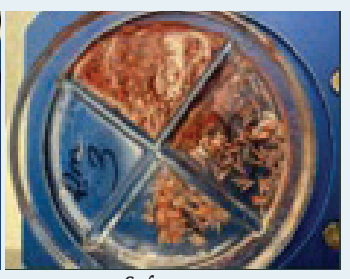

Safe zone

\section{New Strategies for Strategic Graft Distribution}

How can we avoid creating problems caused by the indiscriminate use of grafts harvested from the intermediate zones? Store the grafts harvested from the upper intermediate zone, from the safe zone, and from the lower intermediate zone separately. It is important to know the origin of the grafts when placing.

Option 1: If the placement strategy will be to make sure the intermediate zone grafts are randomized and not concentrated in an area, then the grafts from the three zones need to be thoroughly mixed according to size prior to placement.

Option 2: Keep the grafts separated by zone of origin. Count the total number of grafts of each size from each zone and place the grafts as randomly as possible in ratio of the count per zone. For example, 300 singles came from the safe zone, 100 from the upper intermediate zone, and 100 from the lower intermediate zone. The ratio of placement would be 3:1:1 throughout the targeted recipient area.

Option 3: If the placement strategy is to use the intermediate zone grafts in locations where their early loss would allow the remaining hair to appear natural in distribution, do the following:

- Place the grafts from the upper intermediate zone into the center of the crown or rear of the mid-scalp, if not transplanting the crown.

- Place the grafts from the lower intermediate zone along the frontal and temporal hairlines.

In both instances above, premature loss of hair production by these grafts will appear as natural progression and aging.

\section{Harvesting and Placing in Batches}

Many experienced practitioners harvest and place grafts in batches. Almost always, the grafts in a batch are implanted adjacent to one another in a portion of the recipient area. This presents a special risk when a batch of grafts has been harvested predominantly or exclusively from an intermediate zone and then implanted in proximity to one another in the recipient area. There is a risk that a bald patch may develop later.

\section{Case Studies}

Case 1: Grafts harvested from the lower intermediate zone were placed along the front edge of the hairline and temples in a 24-yearold. Grafts from the safe zone were placed farther back in the hairline zones (Figure 12). If the intermediate zone grafts do stop producing hair prematurely, the hairline will simply recede to a somewhat deeper but natural level.

Case 2: Grafts harvested from the lower intermediate zone were placed in the hairline and temples in a 25 -yearold male. His balding started at age 17 , and he has a family history with many male relatives with class $6 / 7$ baldness. He is taking finasteride and was informed of the need to continue this indefinitely not only to prevent or delay developing class $6 / 7$ baldness but also to maintain hair growth from these grafts. However, we cannot be assured that he will use the finasteride long term. He may have side effects or fear side effects, or he may simply abandon the finasteride. If he discontinues finasteride therapy, it is likely that
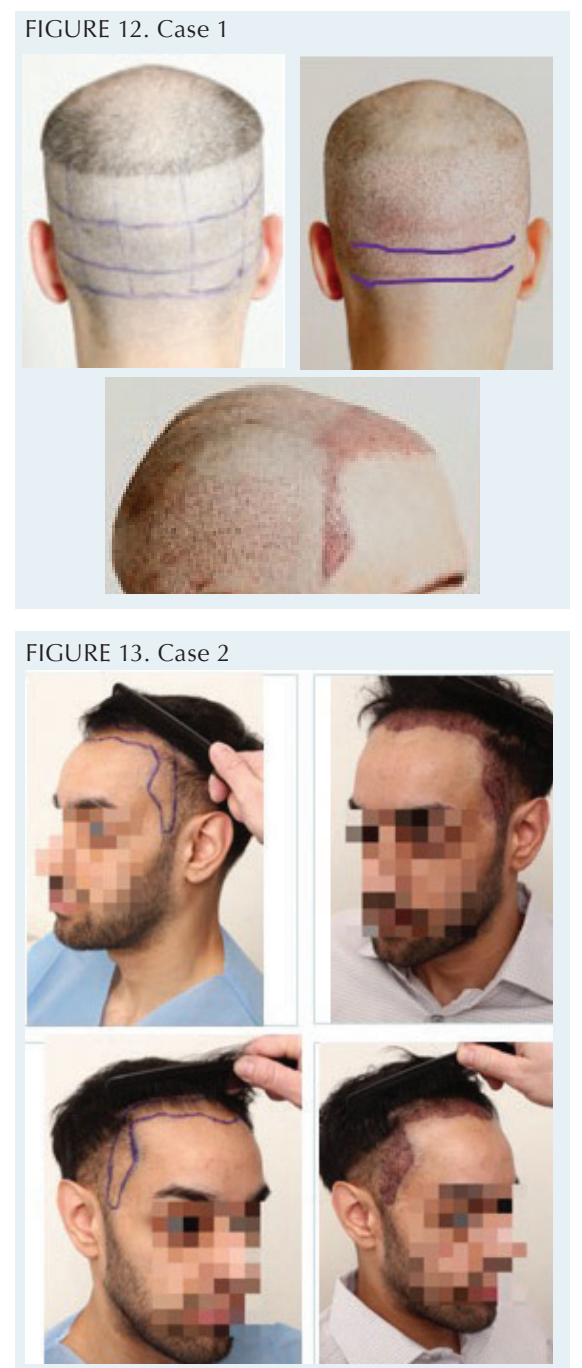


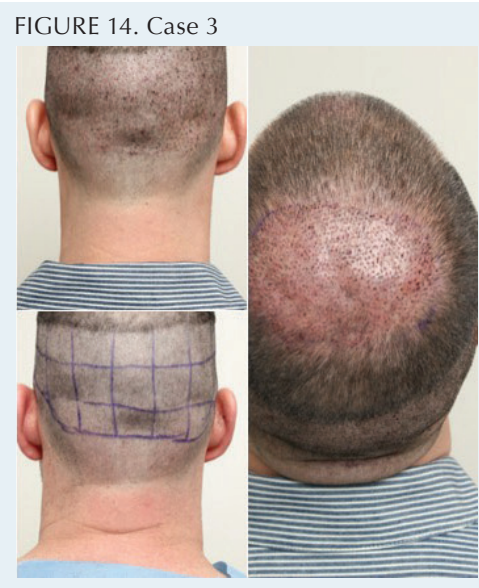
ef probable progression of balding and the need to do additional transplantation to keep up with the enlarging crown balding. As an alternative, grafts were harvested from the upper intermediate zone and transplanted to the center of the vertex and grafts from the safe zone to the periphery of the vertex. If the upper intermediate zone undergoes balding, the grafts from this area will stop producing hair and he will be left with a bald area in the center of the crown that will appear natural. The safe zone donor will have been preserved to connect the vertex periphery to the receding fringe (Figure 14).

\section{CONCLUSION}

The benefits of adopting these strategies will not be known for years. Strategies in graft placement from outside the safe zone are proposed to avoid potential cosmetic problems over time. Routine use of finasteride and minoxidil is recommended when grafts are known to have been harvested from the intermediate zones.

\section{References}

1. Devroye J. Overview of the Donor Area: Basic Principles. In: Unger W, Shapiro R, et al., eds. Hair Transplantation, 5th Ed. Informa Health Care: New York, New York; 2011, pp. 257-260.

2. Yrariat S. Hair Transplantation in Scarring Alopecia. In: True RH, Garg S, Garg AK, eds. Practical Guide to Hair TransplantationInteractive Study for the Beginning Practitioner. Delhi, India: Thieme Med and Scientific Publishers; 2021, pp. 532-534.

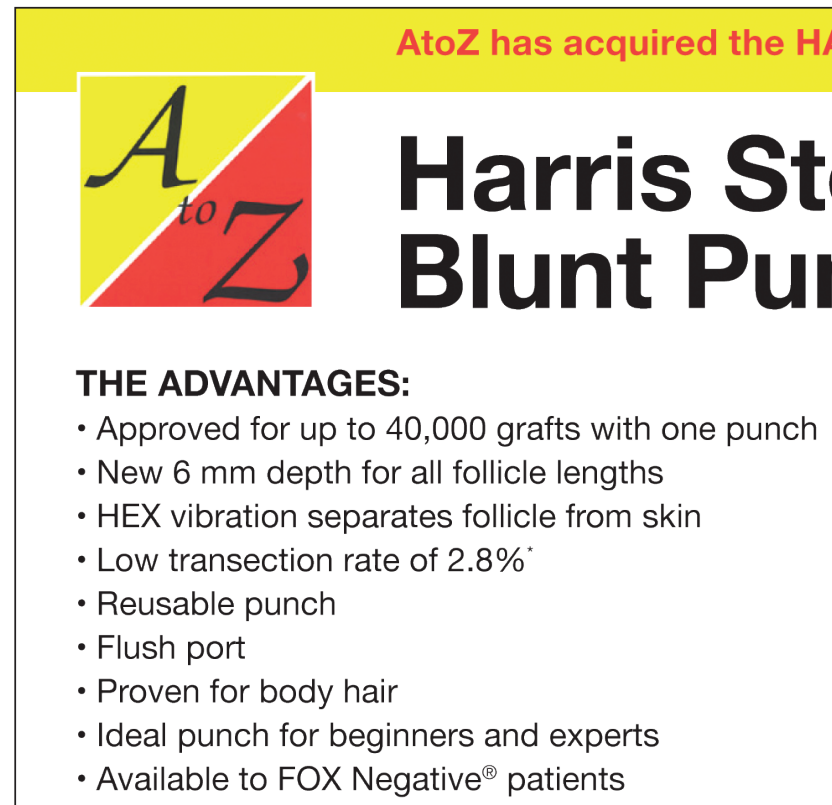

Fast and Efficient Graft Dissection

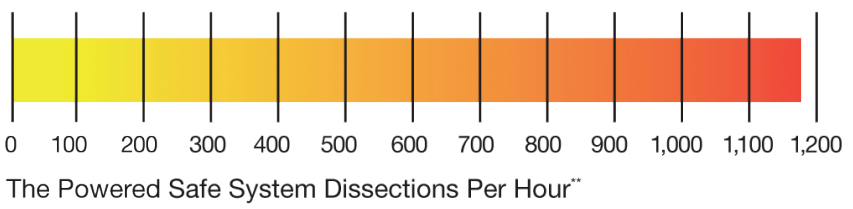

\section{To order, contact us:}

Kenny Moriarty, VP

Email: kenny@georgetiemann.com

Visit us online:

www.georgetiemann.com | www.atozsurgical.com

* In a clinical study by Dr. Harris in over 150 patients and more than 100,000 harvested grafts. General user transection rates may differ.

** Depending on follicular unit configuration and skin characteristics graft dissection rates up to 1200 grafts/hour are possible. Numbers based upon Jim A. Harris, MD extraction rates 